\title{
ORIGINAL
}

\section{Regeneration of caudate lobe after living donor liver transplantation : Comparison with a surrogate model of left lobe graft}

\author{
Yu Saito MD, PhD, FACS'1), Satoru Imura MD, PhD, FACS'1), Yuji Morine MD, PhD, FACS'1), \\ Tetsuya Ikemoto MD, PhD, FACS ${ }^{1}$, Shinichiro Yamada MD, PhD, FACS ${ }^{1)}$, and Mitsuo Shimada MD, PhD, FACS ${ }^{1)}$ \\ ${ }^{1}$ Department of Surgery, Tokushima University, Tokushima, Japan
}

\begin{abstract}
Background : The aim of this study is to clarify the regeneration of the CL (caudate lobe) without any reconstructions of short hepatic veins (SHVr) after LDLT (living donor liver transplantation) and compare the regeneration of the CL after right hepatectomy (Rt. Hx), as the surrogate model of extended left lobe graft (Ex LLG) with complete SHVr. Methods : Eleven Ex LLGs with CL were included in this study. SHVr was not performed in all cases. The volumetry was performed before, one month and six months after LDLT. Seven patients who underwent Rt. Hx were also included in this study as the surrogate model. Results : In Ex LLGs with CL, the regeneration rate of the large CL $(>30 \mathrm{ml})$ was worse than that of small $\mathrm{CL}(<30 \mathrm{ml})$. In the surrogate model, the regeneration rate of the $C L$ was not worse than other segments. However, the regeneration rate of the large $C L$ was also worse than that of small CL even in the presence of complete SHVr. Conclusions : The regeneration of the large CL was worse than that of the small CL regardless of the presence or absence of SHVr, indicating that SHVr in Ex LLG with CL might not be necessary. J. Med. Invest. 68:330-333, August, 2021
\end{abstract}

Keywords : caudate lobe, short hepatic vein, living donor liver transplantation

\section{INTRODUCTION}

First of all, the size of an extended left lobe graft (Ex LLG) more than doubles one month after transplantation (1), and functions as a graft for a recipient for life. However, Ex LLG has more frequent risk of small-for-size-graft (SFSG) than right lobe graft. SFSG sometimes causes graft failure after living donor liver transplantation (LDLT). However, RLG imposes a greater surgical risk on living donors. As a method of increasing the graft volume in the case of SFSG in LDLT, the Shinshu group already reported caudate lobe (CL) transplantation with an extended left lobe graft (Ex LLG) was an innovative and promising method for increasing the graft volume in LDLT (2). We have also already reported a new technique for left lobe graft harvesting proved a promising approach to gain additional volume (2). However, little is known about the fate of the CL after transplantation. As a preliminary report regarding the changes in the CL after transplantation, we already reported that regeneration rate of CL was worse than that of left lobe (LL) at one month after LDLT without any reconstructions of short hetiv veins (SHVr), which are drainage veins of CL that flow directly into inferior vena cava, however, the CL volume increased in almost all patients (1). On the other hand, it was also reported that CL could regenerate proportionally with the LL by complete SHVr (4). It means that the necessity of SHVr in Ex LLG with CL is still controversial.

As our new idea in the present study, we focused on patients after right liver resection (Rt. Hx) for liver tumors. Since CLs of those patients were remnant with $\mathrm{SHVs}$ as drainage veins, the patient with Rt. Hx could be used as the surrogate model of Ex LLG with complete SHVr.

\section{Abbreviations :}

small-for-size grafts ; SFSG, living donor liver transplantation ; LDLT, caudate lobe ; CL, extended left lobe graft ; Ex LLG, left lobe ; LL, reconstructions of short hetiv vein; SHVr, multi-detector computed tomography ; MDCT, standard liver volume; SLV, graft volume; GV, liver volume; $\mathrm{LV}$
Accordingly, we herein clarify the regeneration of CL in case of Ex LLG without SHVr after LDLT and compare the regeneration of CL after Rt. Hx, as the surrogate model of Ex LLG with complete SHVr.

\section{METHODS}

\section{Ethical permission}

This study was approved by The Tokushima University Hospital Ethics Committee and the corresponding regulatory agencies (Tokushima Clinical Trial Management, Approved No 3215) and all the experiments were carried out in accordance with the approved guidelines. Meanwhile, all the patients involved in the study signed the informed consent form and agreed to participate.

\section{Patients}

LDLT ; From February, 2005, to March, 2015, eleven Ex LLG with CL were included in this study. All LDLT operations were performed at Tokushima University Hospital. In all cases, SHVr did not be performed. Detailed operative techniques in LDLT were described in previous report (2).

Rt. Hx ; From December, 2005, to April, 2015, seven patients with initial Rt. Hx were included in this study (five patients with liver metastasis of colorectal cancer with no chemotherapy and two patients with liver abscess or hemangioma). These patients were used for the surrogate model of Ex LLG with complete SHVr.

Received for publication April 5, 2021 ; accepted July 30, 2021.

Address correspondence and reprint requests to $\mathrm{Yu}$ Saito, MD, $\mathrm{PhD}$, Department of Surgery, Tokushima University, 3-18-15 Kuramoto-cho, Tokushima, 770-8503, Japan and Fax : +81-88-631-9698. 


\section{CT volumetry}

3D-reconstruction of the hepatic vasculature was made using data from a contrast enhanced multi-detector computed tomography (MDCT) and SYNAPSE VINCENT software (Fuji Film Medical Co. Ltd., Tokyo, Japan). The volumetry of each segment was performed by "portal segmentation function" of SYNAPSE VINCENT. The volumetry in LDLT was performed before LDLT from the donors and 1 month and 6 months after LDLT (Figure 1). In the surrogate model, the volumetry was performed as well before operation and 1 month and 6 months after operations.

Standard liver volume (SLV), graft volume $(G V)$, and $G V / S L V$ estimation

SLV was calculated according to the formula described by Urata et al. (5). All GV in this study was calculated by volumetry using portal segmentation function.

\section{Regeneration rate}

The regeneration rate was calculated as described below ; (Postoperative liver volume (LV) - Preoperative liver volume) / Preoperative liver volume $\times 100(\%)$

\section{Statistical Analysis}

All results were presented as mean $\pm \mathrm{SD}$. Comparisons between the two groups were performed using a Chi-square test using statistical software (JMP 8.0.1., SAS Campus Drive, Cary, $27513 \mathrm{NC}, \mathrm{USA}$ ). A $p$-value of less than 0.05 was considered statistically significant.

\section{RESULTS}

Increasing the graft volume with $C L$

The LL graft volume was $456 \pm 117 \mathrm{ml}$ at pre LDLT, $1,023 \pm 213 \mathrm{ml}$ at one month post LDLT and $1,175 \pm 275 \mathrm{ml}$ at six months post LDLT, corresponding to a $40 \%$, $89 \%$ and $105 \%$ increase respectively in GV/SLV (Table 1).

The addition of the CL increased the graft volume by $30 \pm 11$ $\mathrm{ml}$ at one month post LDLT and $28 \pm 13 \mathrm{ml}$ at six months post LDLT, corresponding to a $2.6 \%$ and $2.5 \%$ increase respectively in GV/SLV (Table 1).

\section{The regeneration rate of $C L$ in $L D L T$ without $S H V r$}

The regeneration rate of CL was $18 \pm 42 \%$ at one month post LDLT and $0 \pm 42 \%$ at six months post LDLT (Figure 2). 3 cases showed $5 \%$ or more of volume decrease after LDLT, and their preoperative volume were all over $30 \mathrm{ml}$ in volume (data were not shown). Regeneration rate of the small CL (volume $\leq 30 \mathrm{ml}$ ) was $39 \pm 42 \%$ at one month post LDLT and $35 \pm 42 \%$ at six months

Table 1. The regeneration of CL and LL

\begin{tabular}{lcccccc}
\hline & \multicolumn{3}{c}{ Caudate lobe } & \multicolumn{3}{c}{ Left lobe } \\
\cline { 2 - 7 } & Pre & $1 \mathrm{~m}$ & $6 \mathrm{~m}$ & Pre & $1 \mathrm{~m}$ & $6 \mathrm{~m}$ \\
\hline GV (ml) & 28 & 30 & 28 & 456 & 1023 & 1175 \\
GV/SLV (\%) & 2.5 & 2.6 & 2.5 & 40 & 89 & 105 \\
\hline
\end{tabular}

GV, graft volume; SLV, standard liver volume ; m, month(s).

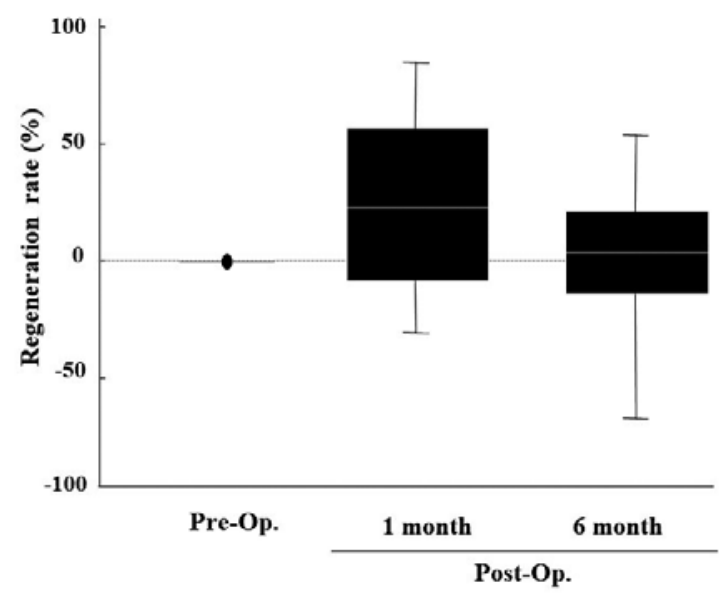

Figure 2. Regeneration rate of CL in LDLT without SHVr The regeneration rate of CL was $18 \pm 42 \%$ at one month post LDLT and $0 \pm 42 \%$ at six months post LDLT.
Pre-Op.

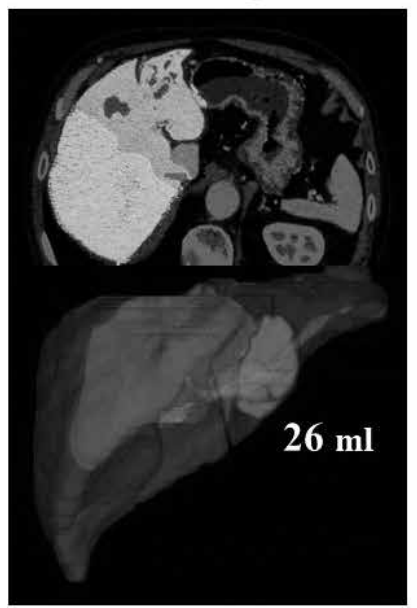

1 month post Op.

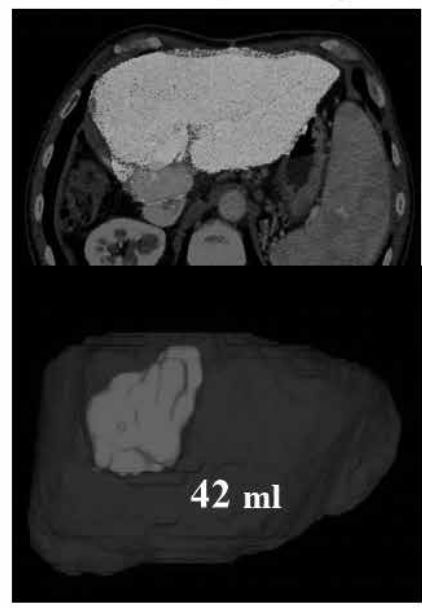

6 months post Op.

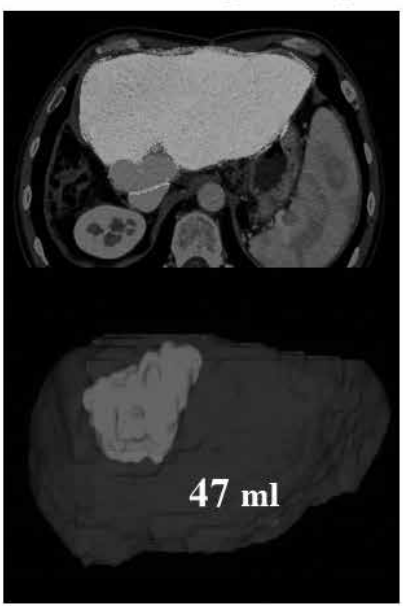

Figure 1. Volumetry of CL

The volumetry of CL was performed by "portal segmentation function" of SYNAPSE VINCENT before LDLT from the donors and 1 month and 6 months after LDLT. 
post LDLT. On the other hand, regeneration rate of the large CL (volume $>30 \mathrm{ml}$ ) was $-27 \pm 18 \%$ at one month post LDLT and $-54 \pm 36 \%$ at six months post LDLT (Figure 3 ). It was suggested that the large CL had worse regeneration than the small CL in Ex LLG without SHVr.

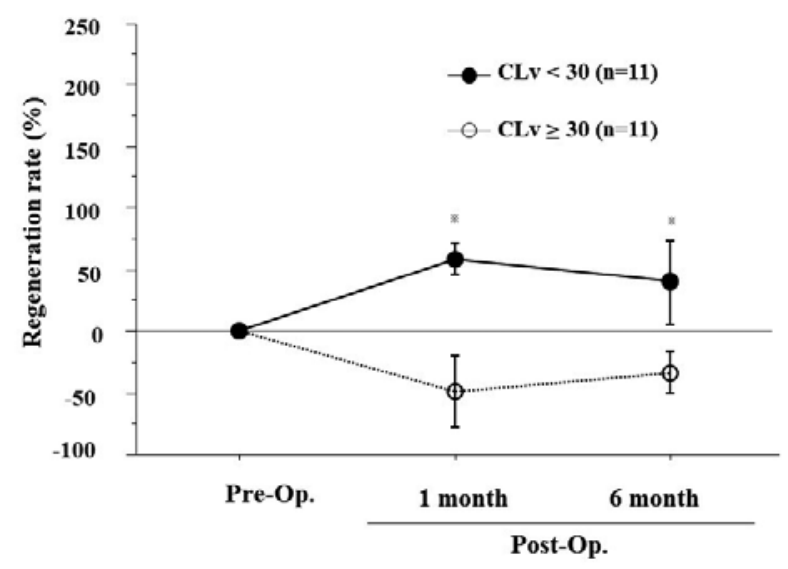

Figure 3. Comparison of the regeneration between small and large CL in LDLT without SHVr

Regeneration rate of the small CL (volume $\leq 30 \mathrm{ml}$ ) was $39 \pm 42 \%$ at one month post LDLT and $35 \pm 42 \%$ at six months post LDLT. On the other hand, regeneration rate of the large CL (volume $>30 \mathrm{ml}$ ) was $-27 \pm 18 \%$ at one month post LDLT and $-54 \pm 36 \%$ at six months post LDLT.

The regeneration rate of $C L$ in in the surrogate model with complete SHVr

The regeneration rate of CL was $49 \pm 62 \%$ at one month post operation and $113 \pm 93 \%$ at six months post operation (Figure 4 ). Regeneration rate of the small CL (volume $\leq 30 \mathrm{ml}$ ) was $56 \pm 69$ $\%$ at one month post operation and $164 \pm 63 \%$ at six months post operation. On the other hand, regeneration rate of the large CL (volume $>30 \mathrm{ml}$ ) was $17 \pm 3 \%$ at one month post operation and $12 \pm 8 \%$ at six months post operation (Figure 5 ). It was suggested that the large CL had also worse regeneration than the small CL in the surrogate model with complete SHVr.

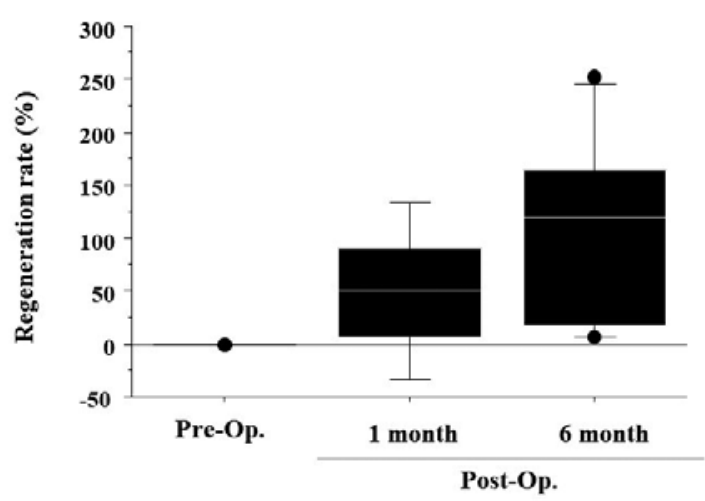

Figure 4. Regeneration rate of CL in the surrogate model with $\mathrm{SHVr}$ The regeneration rate of CL was $49 \pm 62 \%$ at one month post operation and $113 \pm 93 \%$ at six months post operation.

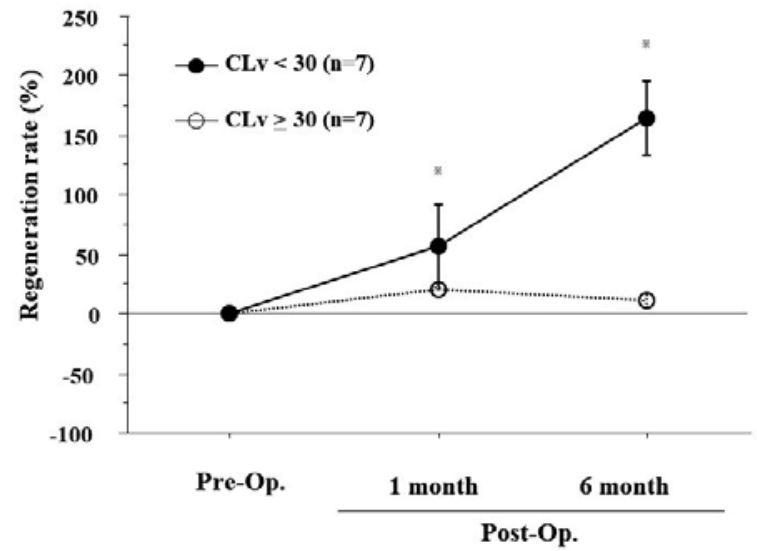

Figure 5. Comparison of the regeneration between small and large $\mathrm{CL}$ in the surrogate model with $\mathrm{SHVr}$

Regeneration rate of the small CL (volume $\leq 30 \mathrm{ml}$ ) was $56 \pm 69 \%$ at one month post operation and $164 \pm 63 \%$ at six months post operation. On the other hand, regeneration rate of the large CL (volume $>30 \mathrm{ml}$ ) was $17 \pm 3 \%$ at one month post operation and $12 \pm 8 \%$ at six months post operation.

\section{DISCUSSION}

The necessity of SHVr in Ex LLG with CL is still controversial. In the present study, we clarified that the regeneration of CL in case of Ex LLG without SHVr after LDLT and compare the regeneration of $\mathrm{CL}$ after $\mathrm{Rt}$. $\mathrm{Hx}$, as the surrogate model of Ex LLG with complete SHVr. It was the first report using the surrogate model of Ex LLG with complete SHVr.

Various CL venous reconstruction techniques were described as one of the feasible solutions to overcome SFSG (6-11). Although the CL volume is small, it is important when the graft volume is critical. We have already showed that the regeneration rate of the transplanted CL and other left lobe graft segments. The regeneration rate of the CL one month after transplantation was smaller ( $62 \pm 24 \%$ ) than that of other left lobe graft segments (152 $\pm 35 \%)$ (3). It was already reported that with reconstruction of the inflow (6) or outflow (7), the regeneration rate of the CL was noted to be equal to or more than that of the other left lobe graft segments. The additional functional volume afforded by CL venous reconstruction might provide an additional safety margin.

On the other hand, Mikami K et al. (12) reported that the GV/SLV ratio of the whole grafts after LDLT showed no difference regardless of the presence or absence of SHVr. In the present study, in the surrogate model of Ex LLG with complete $\mathrm{SHVr}$, regeneration rate of the CL was not worse than other segments. However, regeneration rate of large CL (volume $>30 \mathrm{ml}$ ) had worse than that of the small CL (volume $<30 \mathrm{ml}$ ) as well as LDLT without SHVr cases. It was suggested that the size of CL seemed to be important for regeneration as a new finding.

As limitations of this study, the present study was a retrospective cohort from a single institution. Although it was necessary to add more patients in the present study, the chance of operation of open $\mathrm{Rt}$. Hx with normal liver function have recently decreased. A prospective randomized trial with more patient number will be necessary in the future.

In conclusion, Ex LLG with CL was a useful method for increasing the graft volume. The regeneration of the large CL was worse than that of the small CL regardless of the presence or absence of SHVr. It was suggested that SHVr in Ex LLG with 
CL might be not necessary and the graft size might be important for CL regeneration.

\section{COI DISCLOSURE}

All authors had no sponsorship or funding arrangements relating to this study.

\section{REFERENCES}

1. Ikegami T, Nishizaki T, Yanaga K, Shimada M, Kakizoe S, Nomoto K, Hiroshige S, Sugimachi K: Changes in the caudate lobe that is transplanted with extended left lobe liver graft from living donors. Surgery 129(1) : 86-90, 2001

2. Miyagawa S, Hashikura Y, Miwa S, Ikegami T, Urata K, Terada M, Kubota T, Nakata T, Kawasaki S : Concomitant caudate lobe resection as an option for donor hepatectomy in adult living related liver transplantation. Transplantation 66 : 661-3, 1998

3. Imura S, Shimada M, Miyake K, Ikemoto T, Morine Y, Yoshizumi T: A new technique to acquire additional liver volume for left lobe graft in living donor liver transplantation. Hepatogastroenterology 55(85) : 1206-10, 2008

4. Sugawara Y, Makuuchi M, Takayama T : Left liver plus caudate lobe graft with complete revascularization. Surgery 132(5) : 904-5, 2002 ; author reply 905-6

5. Kawasaki S, Makuuchi M, Matsunami H, Hashikura Y, Ikegami T, Chisuwa H, Ikeno T, Noike T, Takayama T,
Kawarazaki H : Preoperative measurement of Segmental liver volume of donors for living related liver transplantation. Hepatology 18: 1115-20, 1993

6. Kokudo N, Sugawara Y, Kaneko J, Imamura H, Sano K, Makuuchi M : Reconstruction of isolated caudate portal vein in left liver graft. Liver Transpl $10: 1163,2004$

7. Hashimoto T, Sugawara Y, Tamura S, Kaneko J, Motomura N, Takamoto S, Makuuchi M : One orifice vein reconstruction in left liver plus caudate lobe grafts. Transplantation $83: 225,2007$

8. Takayama T, Makuuchi M, Kubota K, Sano K, Harihara Y, Kawarasaki $\mathrm{H}$ : Living-related transplantation of left liver plus caudate lobe. J Am Coll Surg 190 : 635, 2000

9. Sugawara Y, Makuuchi M, Kaneko J, Ohkubo T, Matsui $\mathrm{Y}$, Imamura $\mathrm{H}$ : New venoplasty technique for the left liver plus caudate lobe in living donor liver transplantation. Liver Transpl 8: 76, 2002

10. Hwang S, Lee SG, Ha TY, Ahn CS, Park KM, Kim KH, Lee YJ, Moon DB, Kim KK, Kim YD : Simplified standardized technique for living donor liver transplantation using left liver graft plus caudate lobe. Liver Transpl 10: 1398, 2004

11. Yamazaki S, Takayama T, Inoue K, Higaki T, Makuuchi $\mathrm{M}$ : Interposition of autologous portal vein graft in left liver transplantation. Liver Transpl $11: 1615,2005$

12. Mikami K, Matsuoka N, Maekawa T, Yamauchi Y, Noritomi T, Hoshino S, Shinohara T, Takahashi Y, Noda N, Yamashita $\mathrm{Y}$ : Impact of short hepatic vein reconstruction in living donor adult liver transplantation using a left liver plus caudate lobe graft. Asian J Surg 33: 8-1, 2010 